\title{
HLA-B27 allele frequency in Sri Lankan patients with spondyloarthritides
}

\author{
S Kidnapillai ${ }^{1}$, N D Sirisena ${ }^{1}$, V H W Dissanayake ${ }^{1,2}$ \\ (Index words: HLA-B27 allele frequency in spondyloarthritides)
}

\begin{abstract}
This preliminary study aims to describe the HLA-B27 allele frequency in Sri Lankan patients with spondyloarthritides (SA). An anonymised database of 373 Sri Lankan patients with SA referred for HLA-B27 testing was retrospectively analysed. Eighty five (22.8\%) patients were positive for the $H L A-B 27$ allele. A male preponderance was observed among the positives. The $H L A-B 27$ allele frequency in this sample of patients with SA was relatively low compared to published studies in other populations. Further research is needed to identify the predominant subtypes of the allele to determine which subtypes are the most prevalent in a larger sample of Sri Lankan patients with SA, and to define their association with the specific types of SA.
\end{abstract}

Ceylon Medical Journal 2016; 61: 71-73

DOI: http://doi.org/10.4038/cmj.v61i2.8287

\section{Introduction}

Major histocompatibility complex (MHC) is encoded by a group of genes that play a crucial role in innate and adaptive immunity in vertebrates [1]. It is located in the short arm of human chromosome 6 , and is categorised into two main classes of molecules, namely MHC class I and class II [1, 2], but other regions have also been described recently [3, 4]. MHC class I and II regions encode the human leukocyte antigen (HLA) proteins, which are associated with antigen presentation to T cells [4]. MHC class I molecules present endogenous peptide fragments to the $\mathrm{T}$ cell receptors (TcR) on CD8+T lymphocytes, and MHC class II molecules present intracellular pathogenic peptides to the TcRs on CD4+T lymphocytes $[1,5]$. Human leukocyte antigen B27 (HLA-B27) is an allelic variant of an HLA class I surface antigen that is present in the B locus of MHC [6]. It is a group of 27 different alleles that code for 23 different proteins with a diverse prevalence among different ethnic and racial groups [7]. These 23 different proteins are named as HLA-B*2701 to B*2723 and they vary from one another by one or more amino acid replacements due to changes in exons 2 and 3 coding for alpha 1 and 2 domains. These subtypes can be further subdivided into more than 130 types (http://www.ebi.ac.uk/ cgi-bin/ipd/imgt/hla/allele.cgi).

$H L A-B * 2705$ is the most prevalent B27 allele which is evidently associated with various spondyloarthritides (SA) that are immune-mediated inflammatory disorders $[7,8]$. This subtype is considered the parent molecule, from which other prototypes appeared [9]. SA occur in $0.1-1.4 \%$ of the population and include conditions such as ankylosing spondylitis (AS), reactive arthritis (RA), psoriatic arthritis (PA), Crohn disease, undifferentiated SA and juvenile-onset spondyloarthritis [10]. About 40$50 \%$ of patients with psoriasis, Crohn's disease and sacroilitis and approximately $90 \%$ of AS patients are known to be $H L A-B 27$ positive [11].

HLA-B27 and MHC genes account for $30 \%$ and 40 $50 \%$ of SA, respectively. Although more than $90 \%$ of Caucasians are $H L A-B 27$ positive, only $1-5 \%$ of them develop the disease. This suggests that there could be other genes that are within or outside the HLA complex, which may be responsible for SA [12]. Although recent research describes some of the non-MHC genes that are believed to be involved in SA, HLA-B27 remains the most reported underpinning genetic factor related to SA [13].

In Sri Lanka, there is paucity of data on the frequency of the HLA-B27 allele. Hence this study aims to assess the frequency of this allele in Sri Lankan patients with possible spondyloarthritides (SA) referred for HLA-B27 testing.

\section{Methods}

The HLA-B27 genotypes detected in 373 SA patients referred from all parts of Sri Lanka for HLA-B27genotyping between June 2011 and February 2014 have been

${ }^{1}$ Human Genetics Unit, Faculty of Medicine, University of Colombo, ${ }^{2}$ Asiri Centre for Genomic and Regenerative Medicine, Asiri Surgical Hospital, Colombo, Sri Lanka.

Correspondence: VHWD, e-mail: <vajirahwd@hotmail.com>. Received 8 October and revised version accepted 12 December 2015.

This is an open-access article distributed under the terms of the Creative Commons Attribution License, which permits unrestricted use distribution, and reproduction in any medium, provided the original author and source are credited. 
maintained in an anonymised database completely delinked from the original patients. This database was retrospectively analysed. The diagnosis of SA had been made by the referring clinicians.

Validated protocol was used for the HLA-B27 genotyping [14]. Genomic DNA was extracted from peripheral blood using Qiagen DNA extraction kit as per the manufacturer's instructions (Qiagen, Germany). Allele specific polymerase chain reaction (AS-PCR) was used as a direct genotyping method based on specific primer recognition of a unique $H L A-B 27$ gene region that covers exon 3 spanning codons 91 to 136 resulting in a 135 base pair (bp) fragment [14]. HLA-B27 specific forward primer E91S (5'-GGGTCTCACACCCTCCAGAAT-3') and $H L A-B$ specific reverse primer E136as (5'-CG GCGGTCCAGGAGCT-3') were used to amplify this exon 3 target. As an internal control, PCO4 (5'-CAA CTT CAT CCACGTTCA CC-3') and GH20 (5'-GAAGAG CCAAGG ACA GGT AC-3') primers that recognise the Human $\beta$-globin gene in exon 3 region were used in the same tube, which gave a 268 bp fragment upon amplification [14]. Blank controls were also run to detect false positive results. The detailed PCR conditions have been described [14]. After PCR amplification, the resulting PCR products were run on a $2 \%$ agarose gel along with a validated negative control, and viewed under ultraviolet illumination.

\section{Results}

Of the 373 cases tested, 85 (22.8\%) were positive for the HLA-B27 allele, and 288 (77.2\%) were negative. A male preponderance was observed among the positive individuals with 70 (82.4\%) patients being males and 15 (17.6\%) females. The mean age of the positive cases was 31 years with a range of 13-68 years.

\section{Discussion}

The findings of this study indicate that the HLA-B27 allele exists in Sri Lankan patients with SA at a frequency of $22.8 \%$. HLA-B27 is widely prevalent worldwide among different ethnic and racial groups. In Scandinavian countries, the frequency of $H L A-B 27$ allele is reported to be high compared to other countries [15]. It has been reported that the incidence of SA is directly proportional to the frequency of HLA-B27 allele. However, it is interesting to note that among different ethnic groups, there is a discrepancy in the frequency and distribution of HLA-B27 allele and its subtypes, and their association with SA [16]. This indicates that SA can also be caused by genetic factors other than HLA-B27. Recently, many nonMHC genes have also been found to be associated with predisposition, for example, interleukin 23 receptor (IL23R), endoplasmic reticulum aminopeptidase 1 (ERAP1), interleukin receptor 1 , type II (IL1R2) and anthrax toxin receptor 2 (ANTXR2) [13]. In the temperate sub-arctic regions, where the frequency of the allele is much higher, the association between the allele and SA is relatively low [16]. In contrast, in the Mediterranean regions where the frequency of this allele is reported to be $6.0-18.0 \%$, its association with SA is between $70 \%-80 \%$. Similarly, in the Gulf region, especially in the United Arab Emirates, the association between the HLA-B27 allele and SA was found to be $56 \%$ among Arab patients with SA and $81 \%$ among Asian patients with SA, whereas in Greece, $80 \%$ of the SA patients were positive for this allele. In Southern India, this allele was seen in $76 \%$ of patients with SA [16]. The relatively low allele frequency (22.8\%) we obtained could probably be due to the sample which consisted of a heterogeneous group of patients where the diagnosis has been made by referring clinicians in various parts of the country. In our study, $82.4 \%$ of the positive individuals were males, and this finding agrees with previous studies which report that SA are predominantly seen in males [17]. Further research to identify the predominant subtypes of this allele to determine their prevalence among Sri Lankan patients with SA would be useful.

\section{Conflicts of interests}

There are no conflicts of interest.

\section{References}

1. Wang T, Tan S, Cai Z. Characterisation and expression of MHC class II alpha and II beta genes in mangrove red snapper (Lutjanus argentimaculatus). Mol Immunol 2015; 68: $373-81$.

2. Janeway CAJ, Travers P, Walport M, Shlomchik MJ. The major histocompatibility complex and its functions. In: Immunobiology: The Immune System in Health and Disease. 5thed. New York: Garland Science, 2001.

3. Gruen JR, Weissman SM. Human MHC class III and IV genes and disease associations. Front Biosci 2001; 6: D960-72.

4. Morris DL, Taylor KE, Fernando MM, et al. Unraveling multiple MHC gene associations with systemic lupus erythematosus: model choice indicates a role for HLA alleles and non-HLA genes in Europeans. Am J Hum Genet 2012; 91: 778-93.

5. Landsverk OJB, Bakke O, Gregers TF. MHC II and the Endocytic Pathway: Regulation by Invariant Chain. Scand J Immunol 2009; 70: 184-93.

6. Khan MA, Ball EJ. Genetic aspects of ankylosing spondylitis. Best Pract Res Clin Rheumatol 2002; 16: 675-90.

7. Ball EJ, Khan MA. HLA-B27 polymorphism. Joint Bone Spine 2001; 68: 378-82.

8. Chatzikyriakidou A, Voulgari PV, Drosos AA. What is the role of HLA-B27 in spondyloarthropathies? Autoimmun Rev 2011; 10: 464-8. 
9. Reveille JD. Major histocompatibility genes and ankylosing spondylitis. Best Pract Res Clin Rheumatol 2006; 20: 601-9.

10. Zambrano-Zaragoza JF, Agraz-Cibrian JM, GonzalezReyes C, Duran-Avelar Mde J, Vibanco-Perez N. Ankylosing spondylitis: from cells to genes. Int $J$ Inflam 2013; 2013: 501653doi: 10.1155/2013/501653.

11. Martin TM, Rosenbaum JT. An update on the genetics of HLA B27-associated acute anterior uveitis. Ocul Immunol Inflamm 2011; 19: 108-14.

12. Diaz-Pena R, Castro-Santos P, Aransay AM, et al.Genetic study confirms association of HLA-DPA1 (*)01:03 subtype with ankylosing spondylitis in HLA-B27-positive populations. Hum Immunol 2013; 74: 764-7.
13. Reveille JD. Genetics of spondyloarthritis[mdash]beyond the MHC. Nat Rev Rheumatol 2012; 8: 296-304.

14. Bon MAM, van Oeveren-Dybicz A, van den Bergh FAJTM. Genotyping of HLA-B27 by Real-Time PCR without Hybridization Probes. Clin Chem 2000; 46: 1000-2.

15. Sieper J, Rudwaleit M, Khan MA, Braun J. Concepts and epidemiology of spondyloarthritis. Best Pract Res Clin Rheumatol 2006; 20: 401-17.

16. Mathieu A, Paladini F, Vacca A, et al. The interplay between the geographic distribution of HLA-B27 alleles and their role in infectious and autoimmune diseases: a unifying hypothesis. Autoimmun Rev 2009; 8: 420-5.

17. Zochling J, Smith EU. Seronegative spondyloarthritis. Best Pract Res Clin Rheumatol 2010; 24: 747-56. 